\title{
Proteome analysis of Mycoplasma fermentans cultured under aerobic and anaerobic conditions
}

\author{
Francesca Benedetti ${ }^{1,2^{*}}$ (D), Selvi Krishnan ${ }^{1}$, Fiorenza Cocchi ${ }^{1,3}$, Hervé Tettelin ${ }^{4,5}$, Robert C. Gallo ${ }^{1,3}$,
} Davide Zella $a^{1,2}$ and Sabrina Curreli ${ }^{1,3}$

\begin{abstract}
Background and aims: Mycoplasmas are ubiquitous pathogens found not only in humans but also in animals, plants, insects and soil. Though they usually grow better in an aerobic environment, mycoplasmas are also facultative anaerobic microorganisms. Following infection, the transition of a microorganism from a normal environment into an anaerobic one (e.g. dead or dying tissue) may result in production of a higher number of bacterial toxins. The resolution of the bacterial proteome during the aerobic/anaerobic switch could thus allow the identification of potential pathogenic determinants and pathways.

Methods: We used two-dimensional gel electrophoresis (2-DE) coupled with matrix-assisted laser desorption/ ionization time-of-flight/tandem mass spectroscopy (MALDI-TOF MS/MS) and subsequent mass spectrometric analysis to characterize the liposoluble and hydrosoluble protein fractions of a strain of Mycoplasma fermentans isolated in our lab (MFI), that was cultured under either aerobic or anaerobic conditions.

Results: We identified the 27 most abundant proteins in the liposoluble fraction and the 30 most abundant proteins in the hydrosoluble fraction and determined their modulation under aerobic and anaerobic growth. By using Protein ANalysis TrougH Evolutionary Relationships (PANTHER) and the Search Tool for the Retrieval of Interacting Genes/Proteins (STRING) software analysis tools, we were able to identify, define and organize the function of each protein, as well as to determine the specific interactome.

Conclusions: Our work provides the first proteome reference map of Mycoplasma fermentans obtained under aerobic and anaerobic growing conditions. These data may help to better understand the mechanisms of pathogenicity of this microorganism and define new diagnostic targets.
\end{abstract}

Keywords: Mycoplasma, Proteomic analysis, Aerobic, Anaerobic, 2D-electrophoresis

\section{Background}

Mycoplasmas are prokaryotic organisms of the class Mollicutes (that literally means "soft skins") [1] which comprises over 200 species, widely spread in nature as obligate parasites of humans, mammals, reptiles, fish, arthropods, and plants [2]. Their cellular membrane contains phospholipids, glycolipids, sterols and various proteins while lacking a rigid cell wall [2].

\footnotetext{
* Correspondence: FBenedetti@ihv.umaryland.edu

${ }^{1}$ Institute of Human Virology, School of Medicine, University of Maryland, Baltimore, MD 21201, USA

${ }^{2}$ Department of Biochemistry and Molecular Biology, School of Medicine, University of Maryland, Baltimore 21201, USA

Full list of author information is available at the end of the article
}

These organisms replicate by binary fission and have limited biosynthetic capabilities, depending mostly on the environment (host or growth medium) for the supply of most nutrients. Most of them exist in fact as parasites with strict host and tissue specificities.

Despite their relatively small physical (150 to 350 $\mathrm{nm})$ and genomic size (600-2200 kb) [3-6], mycoplasmas are considered pathogens and cofactors in diverse diseases [7]. Also, mycoplasmas are known to elicit strong host immune responses due to the presence on their membrane of lipoproteins able to trigger a Toll-like receptor-mediated response [8-12]. The variation in mycoplasma lipoproteins attributed

C The Author(s). 2019 Open Access This article is distributed under the terms of the Creative Commons Attribution 4.0 International License (http://creativecommons.org/licenses/by/4.0/), which permits unrestricted use, distribution, and 
to genome rearrangements and genetic insertions have been demonstrated to lead to phenotypic plasticity that allows also for the evasion of the host's defense system and pathogenesis [13-15]. Some strains of mycoplasma can invade intracellularly [16-18], including Mycoplasma fermentans [19], enhancing their ability to evade the immune system [20]. Given their presence on mostly mucosal surfaces (respiratory and urogenital tracts, eyes, alimentary canal, mammary glands and joints [21-23]), mycoplasmas have been associated to respiratory $[24,25]$, urogenital [26-28] and rheumatoid diseases [29-31].

In general, mycoplasmas are facultative anaerobes, except for Mycoplasma pneumoniae, which is a strict aerobe $[21,32]$. The presence of the anaerobic form of these microorganisms is usually explained by the availability of oxygen and by the colonization of microhabitats protected from air. In fact, mycoplasmas are able to invade the deep tissues as a result of mucosal surface disruption, local trauma, surgery, tissue necrosis and impaired clearance of a sterile site. Their ability to grow in anaerobic environments may lead to localized infections $[33,34]$. To this regard, in a number of cases mycoplasmas are considered causative agents for localized infections, and the difficulty in their isolation likely renders these associations underestimated [35-39].

Data regarding the constitutive proteome in pathogens under relevant conditions can definitely provide valuable information on their metabolic regulation and pathogenesis. In this study, we performed the proteome analysis of a strain of Mycoplasma fermentans MFI, isolated in our laboratory from samples obtained from an HIV-seropositive patient [40]. This strain of Mycoplasma fermentans has a nucleotide sequence very similar to another strain isolated in our lab (MFII) and to the prototypes Mycoplasma fermentans PG18 and M64 [3, 41, 42]. We chose to study the strain MFI because its DnaK showed broad anticancer properties both in vivo and in vitro [40]. To this regard we demonstrated that this mycoplasma promoted lymphomagenesis in an in vivo mouse model and its bacterial chaperone protein, DnaK, reduced the activity of PARP1 and p53, involved in DNA damage control/repair and cell-cycle/apoptosis, respectively [40]. Protein expression profiles of this microorganism cultured in aerobic and anaerobic conditions were determined using two-dimensional gel electrophoresis (2-DE) and proteins were further identified using MALDI-TOF/MS-MS.

The present work provides unique information regarding the proteome of Mycoplasma fermentans MFI isolate grown in two different conditions. Our data may help to identify potential candidates for functional studies and better elucidate pathways related to pathogenesis, as well as possible virulenceassociated diagnostic and therapeutic targets.

\section{Methods}

Bacterial strains and culture conditions

In this study we analyzed the strain of Mycoplasma fermentans MFI isolated in our laboratory from samples of an HIV-infected patient [40]. The whole genome shotgun sequence of strain MFI was obtained with the Roche 454 FLX pyrosequencing technology at $\sim 75 \mathrm{x}$ and $\sim 45 \mathrm{x}$ coverage, respectively. Raw reads were assembled into contigs using Newbler v1.1.03.24 then annotated using the Institute for Genome Sciences automated Annotation Engine pipeline (http://ae.igs.umaryland.edu/). The annotated whole genome sequence has been deposited at the NCBI Whole Genome Shotgun (WGS) repository (MF-I1 - ATFG00000000).

Laboratory isolates of MFI were grown both in anaerobic and aerobic conditions. The anaerobic growth was performed in PPLO medium (BD) supplemented with $17 \%$ heat inactivated fetal bovine serum (ThermoFisher Scientific), tryptone (BD) 1\%, glucose (Sigma-Aldrich) $0.5 \%$, yeast extract solution $3.5 \%$ and yeastolate $1 \%$, at $37^{\circ} \mathrm{C}$ (both from BD). Anaerobic conditions were achieved by growing the cultures inside anaerobic jars containing GasPaks (BD). The aerobic growth was performed in 243 medium containing heart infusion broth (BD Biosciences) supplemented with $20 \%$ heat inactivated horse serum and $10 \%$ yeast extract solution (ThermoFisher Scientific), at $37^{\circ} \mathrm{C}$. Mycoplasma cultures were harvested in late $\log$ phase, collected by centrifugation $(20 \mathrm{~min}$ at 10 , $000 \mathrm{~g}$ at $4{ }^{\circ} \mathrm{C}$ ), and washed three times with PBS. At least three mycoplasma pellets were obtained from each bacterial culture replicate, and used for proteomic analysis.

\section{Protein extraction and triton $\mathrm{X}-114$ fractionation}

For total protein extracts, bacterial pellets were resuspended in $1 \%$ hot SDS, incubated for $3 \mathrm{~min}$ at $95^{\circ} \mathrm{C}$, chilled and diluted with lysis buffer $(10 \mathrm{mM}$ Tris $\mathrm{HCl}$ $\mathrm{pH} 7.4,0.15 \mathrm{M} \mathrm{NaCl}, 1 \mathrm{mM}$ EDTA, PBS, 1\% V/v Triton $\mathrm{X}-114$ and protease inhibitors). Lysates were sonicated for $5 \mathrm{~min}$ on ice-water $\mathrm{mix}$ and insoluble material was removed by centrifugation $\left(30 \mathrm{~min}\right.$ at $10,000 \mathrm{~g}$ at $4{ }^{\circ} \mathrm{C}$ ). Hydrophilic and hydrophobic protein fractions were obtained by Triton X-114 fractionation [43, 44].

\section{Delipidation and protein precipitation by chloroform/ methanol/water}

The method developed from Wessel [44, 45] was used for delipidation and protein precipitation. Delipidated protein pellets were air-dried and resuspended in 
ReadyPrep protein extraction kit from Biorad, following the instructions from the manufacture. Protein samples were quantified using RC DC protein assay kit (Biorad).

\section{D-Dige proteomics profile of mycoplasmas}

We used 2D-Dige analysis followed by MALDI-TOF/ MS-MS protein identification to analyze the proteomic profile of MFI growth in aerobic versus anaerobic conditions. Proteomics profile was performed by Applied Biomics, following a protocol previously published [46]. Briefly, delipidated hydrophilic and hydrophobic protein fractions from MFI growth either in aerobic condition or in anaerobic condition were labeled with $\mathrm{Cy} 3$ or Cy5 dyes, and a 1:1 mix was also labeled with $\mathrm{Cy} 2$ which was included with the test samples as an internal labeling control. Samples were then analyzed using an Amersham Biosciences 2D-gel system (Amersham Biosciences). DIGE images were detected with a Typhoon TRIO scanner, analyzed by ImageQuant software version 5.0 (GE Healthcare) and processed with DeCyder software version 6.5 (GE Healthcare) for image analysis. Student's $t$ test analysis was used for statistical analysis, and significant change of protein abundance was defined as at least 1.5 -fold difference $(P<0.05)$. NCBI and MFI library were used as sequence databases. The detailed protocol of the proteomic analysis procedure is available elsewhere [46].

\section{Functional and biological characterization of the proteins} MS data were subjected to gene ontology analysis with Blast2GO [47] and used to generate tables containing information regarding protein identity, molecular weight, pI (isoelectric point), peptides count, identity score and accession number. Identified proteins were then classified based on cellular components, protein class, biological processes and molecular functions using the PANTHER bioinformatics tool (v. 9.0; http://www. pantherdb.org) [48, 49]. Finally the biological associations among the proteins from the aerobic and anaerobic growth conditions were investigated using the online STRING software (v. 10; https://string-db.org) [50]. A medium confidence score of 0.4 was used as the cutoff criterion.

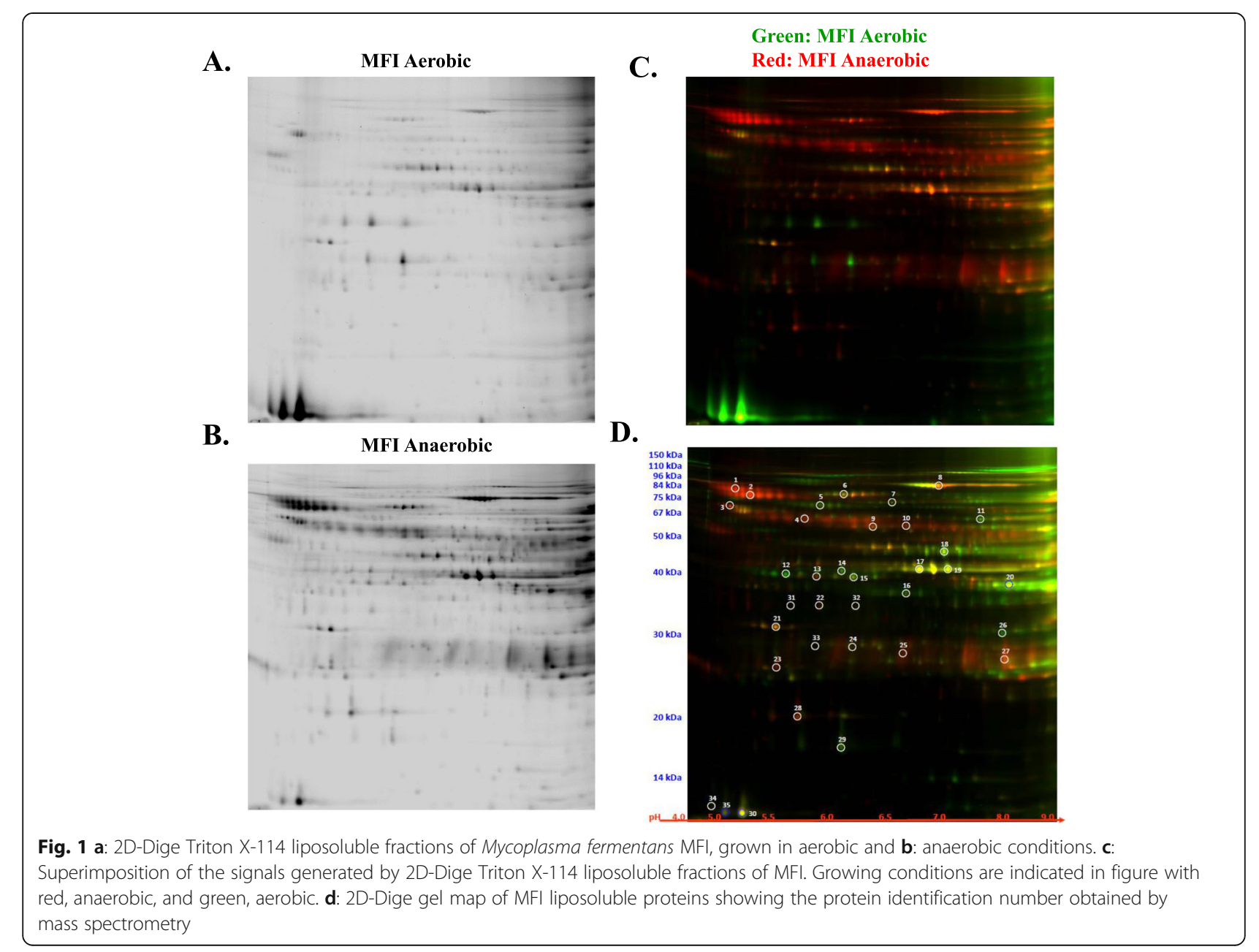




\section{Results}

2D-electrophoresis protein comparison and quantification from Mycoplasma fermentans cultured in aerobic and anaerobic conditions

We used a 2D-Dige electrophoresis to characterize quantitatively and qualitatively the protein profile of Mycoplasma fermentans grown in aerobic and anaerobic conditions. A specific procedure was employed to separate proteins soluble in lipids (liposoluble fraction) or soluble in water (hydrosoluble fraction). The liposoluble (Fig. 1a-c) and hydrosoluble (Fig. 2a-c) fractions from aerobic and anaerobic culture conditions were compared by superimposition of the signals to the corresponding fraction. By using mass spectrometry, we identified the 35 (27 of which were uniquely identified) most abundant proteins differentially expressed in the liposoluble fraction (Table 1) and the 38 (30 of which were uniquely identified) most abundant proteins in the hydrosoluble fraction (Table 2). A few proteins were identified in both fractions, most likely because of a cross-contamination between the two phases due to their abundance. We calculated the ratio of the single protein expression in anaerobic versus aerobic culture conditions (see
Material and Methods). These values are shown in Table 3a (for the liposoluble fraction) and Table $3 \mathrm{~b}$ (for the hydrosoluble fraction). The differential expression of the isolated proteins in anaerobic and aerobic conditions is illustrated in Fig. 3a (for the liposoluble fraction) and Fig. 3b (for the hydrosoluble fraction). The higher ratio of the enzyme arginine deaminase (arcA) in the anaerobic fraction (Fig. 3a) indicated that our experimental procedures were correct, since this enzyme is a repressor of various aerobic enzymes [51].

A remarkable difference between the two isolated fractions consisted in the high protein ratio of anaerobic versus aerobic observed in the liposoluble fraction versus the hydrosoluble (Fig. 3). In particular, we observed minor differences in the hydrosoluble protein ratio between the anaerobic and aerobic grown mycoplasma with an average ratio ranging from -2.58 to 3.53 . In contrast, we saw large differences in the expression of liposoluble proteins in anaerobic versus aerobic, with an average ratio ranging from -21.61 to 8.05 . The homogenous expression in aerobic and anaerobic mycoplasma hydrosoluble proteins suggests
A.

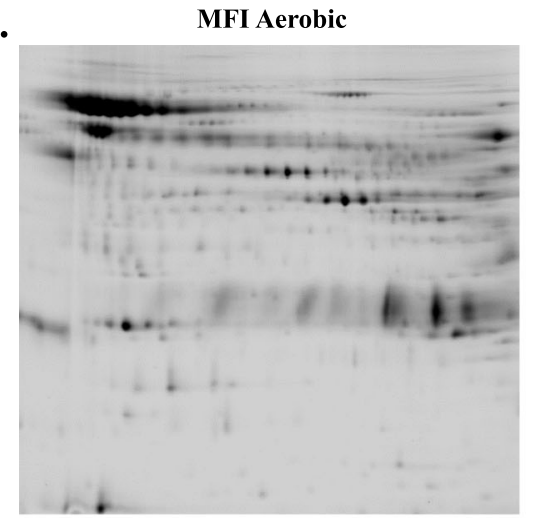

B.

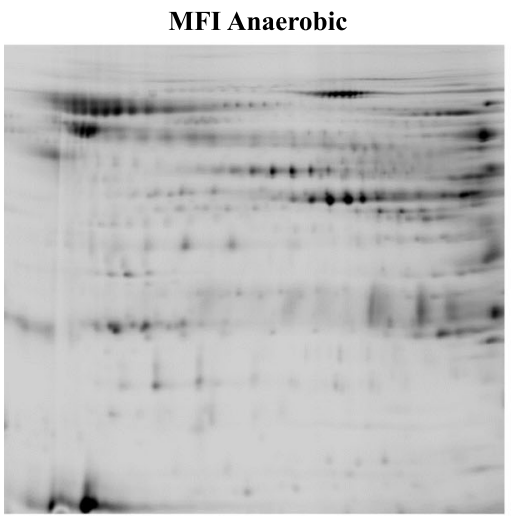

C.

Green: MFI Aerobic

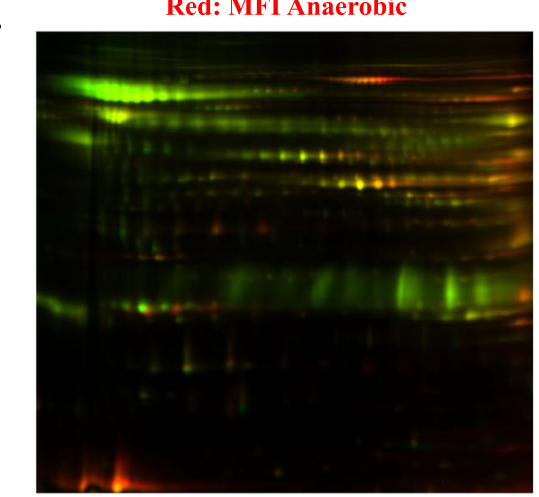

D.

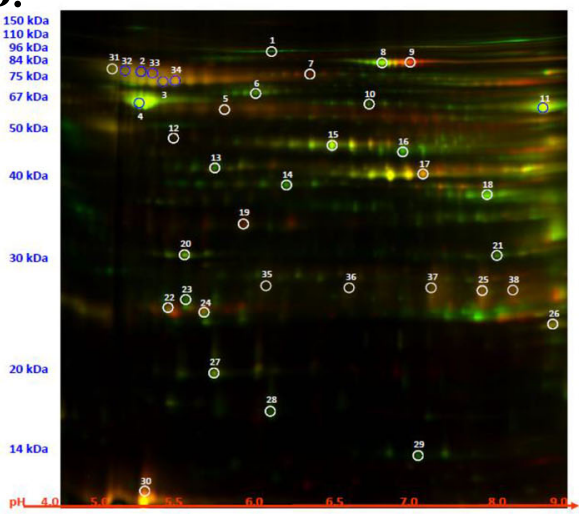

Fig. 2 a: 2D-Dige Triton X-114 hydrosoluble fractions of Mycoplasma fermentans MFI, grown in aerobic and b: anaerobic conditions. c: Superimposition of the signals generated by 2D-Dige Triton X-114 hydrosoluble fractions of MFI. Growing conditions are indicated in figure with red, anaerobic, and green, aerobic. d: 2D-Dige gel map of MFI hydrosoluble proteins showing the protein identification number obtained by mass spectrometry 


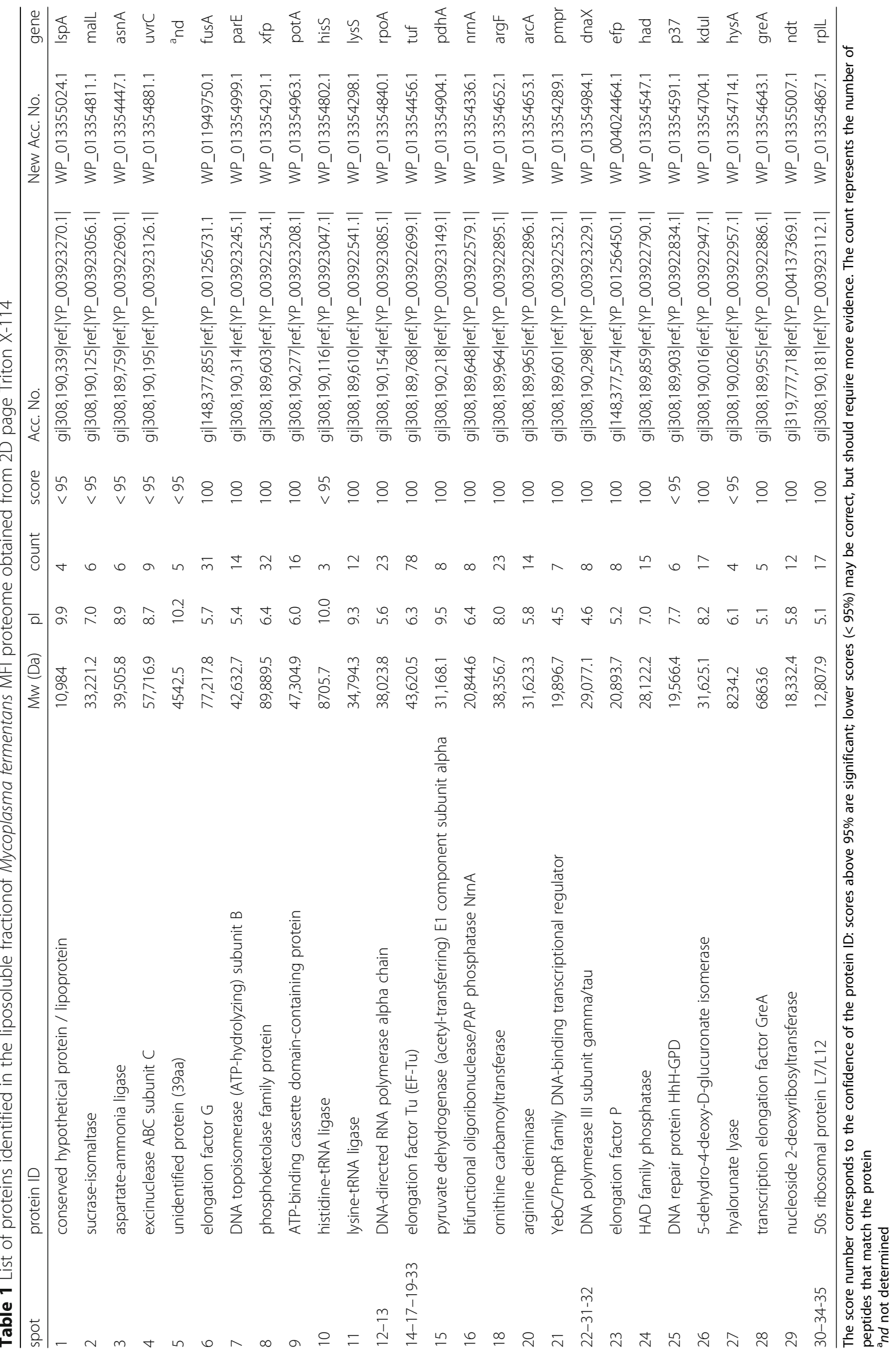




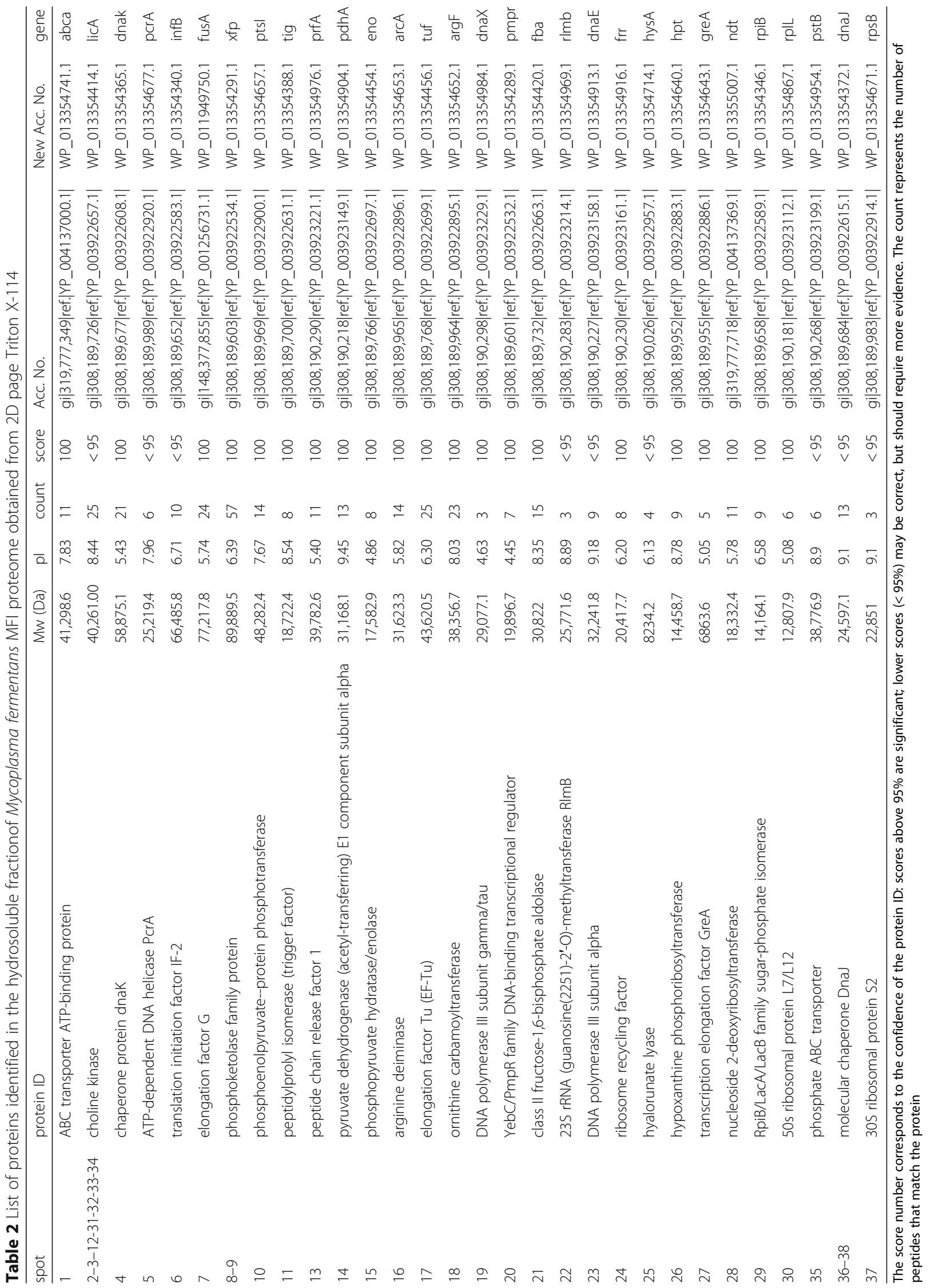


Table 3 Protein ratio of liposoluble (A) and hydrosoluble (B) fraction of Mycoplasma fermentans. The ratio MFI anaerobic versus MFI aerobic is shown

\begin{tabular}{|c|c|c|}
\hline \multicolumn{3}{|c|}{ A. Liposoluble proteins } \\
\hline spot\# & gene & $\mathrm{MFI} A n / \mathrm{MFI} \mathrm{Ae}$ \\
\hline 1 & IspA & 48.6 \\
\hline 2 & malL & 27.89 \\
\hline 3 & asn A & 4.78 \\
\hline 4 & uvrC & 11.46 \\
\hline 5 & *nd & 1.55 \\
\hline 6 & fusA & 2.31 \\
\hline 8 & $x f p$ & 5.59 \\
\hline 9 & potA & 7.4 \\
\hline 10 & hisS & 11.92 \\
\hline 11 & lysS & -1.35 \\
\hline $12-13$ & rpoA & from -1.85 to -1.04 \\
\hline 14-17-19-33 & tuf & from -29.58 to 1.74 \\
\hline 15 & pdhA & 3.22 \\
\hline 16 & nrnA & 1.11 \\
\hline 18 & $\operatorname{argF}$ & 3.29 \\
\hline 20 & $\operatorname{arcA}$ & 2.17 \\
\hline $22-31-32$ & dnaX & from -22.34 to -21.41 \\
\hline 23 & efp & 1.69 \\
\hline 24 & had & -11.04 \\
\hline 25 & p37 & 5.91 \\
\hline 26 & kdul & -1.8 \\
\hline 27 & hysA & 5.56 \\
\hline 28 & greA & 2.72 \\
\hline $30-34-35$ & rplL & from -83.41 to -43.26 \\
\hline \multicolumn{3}{|c|}{ B. Hydrosoluble proteins } \\
\hline spot\# & gene & MFI An/MFI Ae \\
\hline 1 & abca & 1.16 \\
\hline $2-3-12-31-32-33-34$ & licA & from -4.38 to -2.85 \\
\hline 4 & dnak & -1.69 \\
\hline 5 & pcrA & -1.74 \\
\hline 6 & $\operatorname{infB}$ & -1.55 \\
\hline $8-9$ & $x f p$ & from 3.22 to 15.14 \\
\hline 10 & ptsl & 1.01 \\
\hline 11 & tig & 1.3 \\
\hline 13 & prfa & -1.29 \\
\hline 15 & eno & -1.39 \\
\hline 17 & tuf & 3.52 \\
\hline 19 & dnaX & 3.34 \\
\hline 20 & pmpr & 2.11 \\
\hline 21 & $\mathrm{fba}$ & 2.7 \\
\hline 22 & $\mathrm{rlmb}$ & -3.69 \\
\hline
\end{tabular}

Table 3 Protein ratio of liposoluble (A) and hydrosoluble (B) fraction of Mycoplasma fermentans. The ratio MFI anaerobic versus MFI aerobic is shown (Continued)

\begin{tabular}{lll}
\hline 23 & dnaE & -1.33 \\
24 & frr & 4.6 \\
25 & hysA & -2.64 \\
26 & hpt & 4.84 \\
28 & ndt & -1.89 \\
29 & rpiB & 2.6 \\
30 & rplL & 9.82 \\
35 & pstB & -3.12 \\
$36-38$ & dnaJ & from -3.11 to -2.29 \\
37 & rpsB & -2.7 \\
\hline
\end{tabular}

A cut-off value of \pm 1.5 was used to distinguish between proteins similarly expressed in aerobic and anaerobic conditions $(-1.5 \leq$ ratio $\leq+1.5)$ from proteins comparatively more expressed (ratio $>+1.5$ and $<-1.5$ ). When the same protein was found in both fractions, only the spot with higher ratio, consisting with the most represented protein, was included

that this fraction mostly contains proteins essential for the life of the bacteria, while the substantial changes in the expression of liposoluble proteins in anaerobic versus aerobic, indicates that this fraction includes proteins necessary for the growth of mycoplasma in the presence or the absence of oxygen.

\section{Functional classification of proteins}

The identified proteins from anaerobic and aerobic growth were classified using the PANTHER software according to the Gene Ontology (GO) annotations for protein classes and molecular functions [48, 49] (Fig. 4 and Additional file 1: Table S1). Mycoplasma genitalium was the only mycoplasma specie annotated in PANTHER, therefore it was used as a reference for these classifications.

When the proteins were classified according to their class (Fig. 4a-b), the dominant class for both culture conditions consisted in the nucleic acid binding proteins (Fig. 4a 46.7\% anaerobic and Fig. 4b 40\% aerobic, respectively). A striking difference between the two culture conditions was that proteins with isomerase activity were only found in mycoplasma grown in anaerobic conditions (12\%) (Fig. 4a). The group of proteins with isomerase activity included DNA topoisomerase, rpiB family of sugar-phosphatase isomerase and hypoxantine phosphoribosyltransferase (indicated as parE, rpiB and hpt, respectively) (Additional file 1: Table S1). Of these isomerases, rpiB and hpt are metabolic enzymes involved in the pentose phosphate pathway of Carbohydrate degradation and the Purine metabolism, respectively.

When proteins were classified according to their molecular functions, most of the proteins from both culture conditions were largely involved in catalytic activities: $38.9 \%$ in anaerobiosis (Fig. 4c) and $35.7 \%$ in aerobiosis 


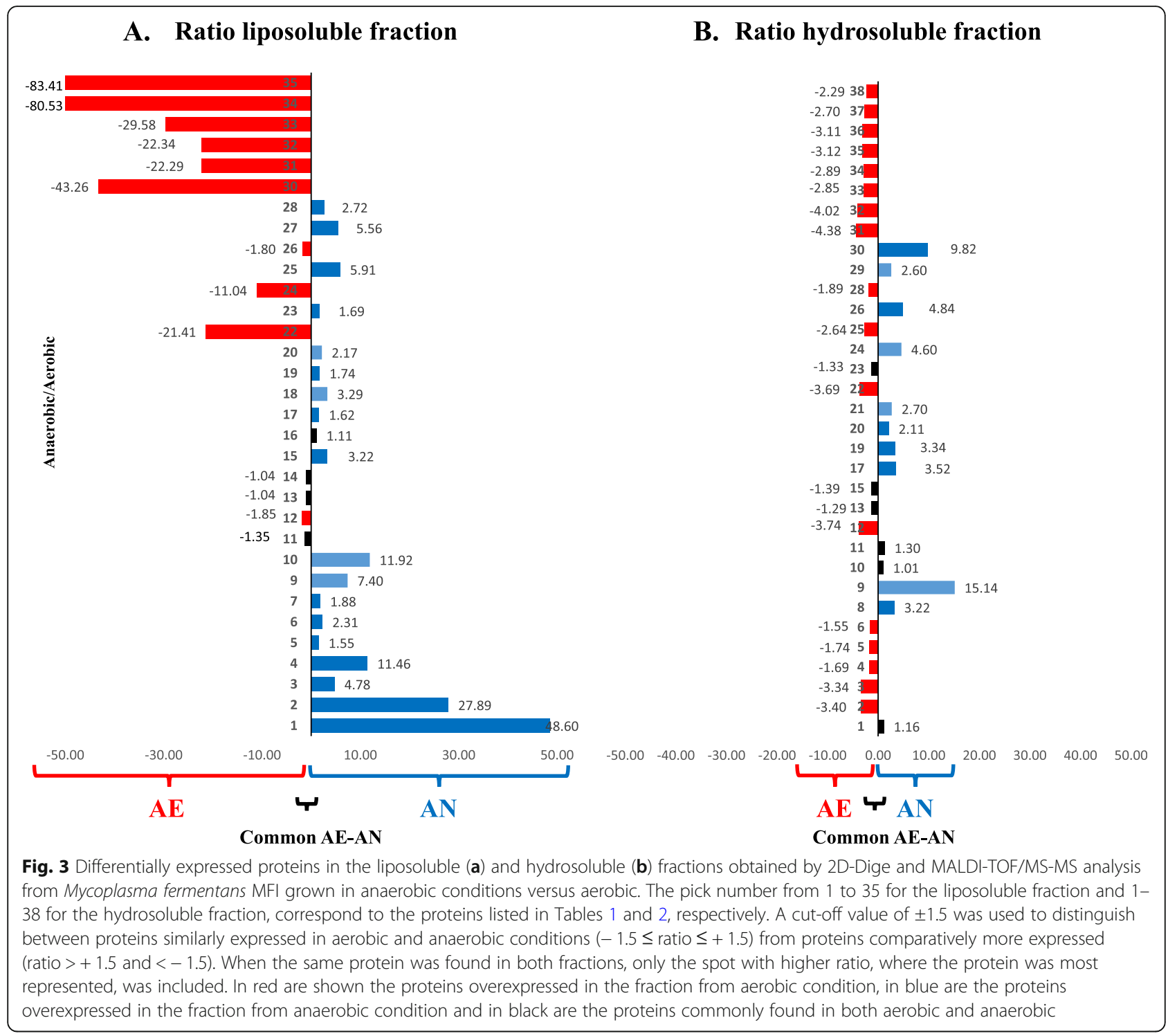

(Fig. 4d) (Additional file 1: Table S1). The amount of proteins with translation regulatory activity was higher in anaerobic mycoplasma ( $16.7 \%$ versus $7.1 \%$ in aerobic), while the proteins with structural activity were expressed at higher percentage in the aerobic mycoplasma (14.3\% versus $5.6 \%$ in anaerobic) (Fig. 4c).

Despite the similarity in protein class and function observed from the PANTHER output (Fig. 4), different sets of proteins were specifically expressed in anaerobic as well in aerobic MFI (Additional file 1: Table S1).

\section{Biological association among proteins}

A major limitation of the PANTHER analysis was that numerous proteins from Mycoplasma fermentans were not found in the reference genome of Mycoplasma genitalium (see legend Additional file 1: Table S1). As a consequence, we were unable to predict metabolic pathways with PANTHER. Despite this limitation, we searched the STRING database [50] for the biological associations and network of the proteins belonging to the two different culture conditions (anaerobic and aerobic). Mycoplasma hominis was used as the reference genome. A protein-protein interaction network of the identified proteins is shown in Fig. 5. Table 4 indicates the biological processes, molecular function and Kyoto Encyclopedia of Genes and Genomes (KEGG) pathways of protein-protein interaction. The String database identified 4 KEGG pathways upregulated in the anaerobic mycoplasma and 3 KEGG pathways in the aerobic growth (Table 4). Specifically, we found that the culture of anaerobic MFI was enriched in metabolic pathways, biosynthesis of secondary metabolites and the RNA polymerase pathways. In contrast, the ribosome and the mismatch repair pathways were enriched in the aerobic 


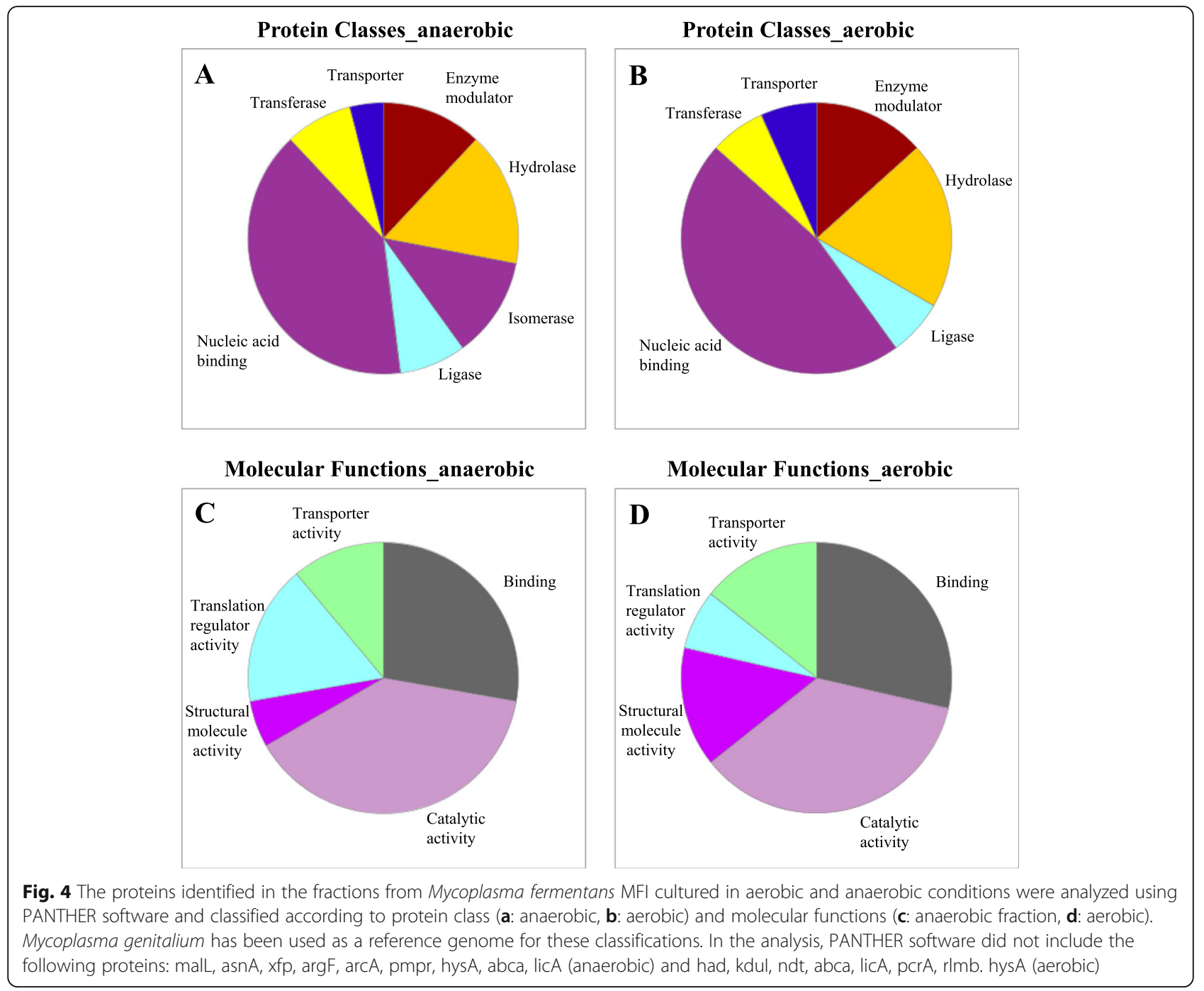

MFI. These results suggests that compared to the mycoplasma grown in aerobic conditions, various metabolic pathways are upregulated by mycoplasma in the absence of oxygen, while the purine metabolism was upregulated in both growth conditions.

Some proteins from anaerobic MFI, not comprised in the STRING analysis, were manually searched in Uniprot (https://www.uniprot.org) and included: argF that is part of the biosynthesis of secondary metabolites, MaIL and xfp that are enzymes involved in carbohydrate metabolic process, pmpr that is a transcriptional regulator, and the protein component of high-affinity transport system p37. Of note, p37 lipoprotein from Mycoplasma hyorhinis has the ability to promote cancer cell invasiveness and metastasis [52] and in our system it was overexpressed in anaerobic conditions (Table 3 and Fig. 3).

The proteins from aerobic MFI not comprised in the STRING analysis included: had, a DNA replication factor, kdui factor involved in glycan metabolism, pstb transport protein and rlmb, involved in RNA metabolism. Finally, proteins commonly expressed in MFI aerobic and anaerobic not found in STRING included: hisA, involved in carbohydrate metabolic process, nrna nuclease and the transport proteins abca and ptsL.

\section{Discussion}

Mycoplasmas are prokaryotic organisms of the class Mollicutes, widely spread in nature [2]. Because of their limited biosynthetic capabilities, mycoplasmas rely on the environment or host cells for some of their essential metabolic requirements, and can also grow as intracellular parasites exhibiting strict host and tissue specificities $[21,32]$.

Some mycoplasmas have been associated with human cancers, including prostate cancer [53], oral cell carcinoma [54] and non-Hodgkin's lymphoma (NHL) in HIVseropositive subjects [55]. Although their role remains unclear and controversial, it has been shown that 


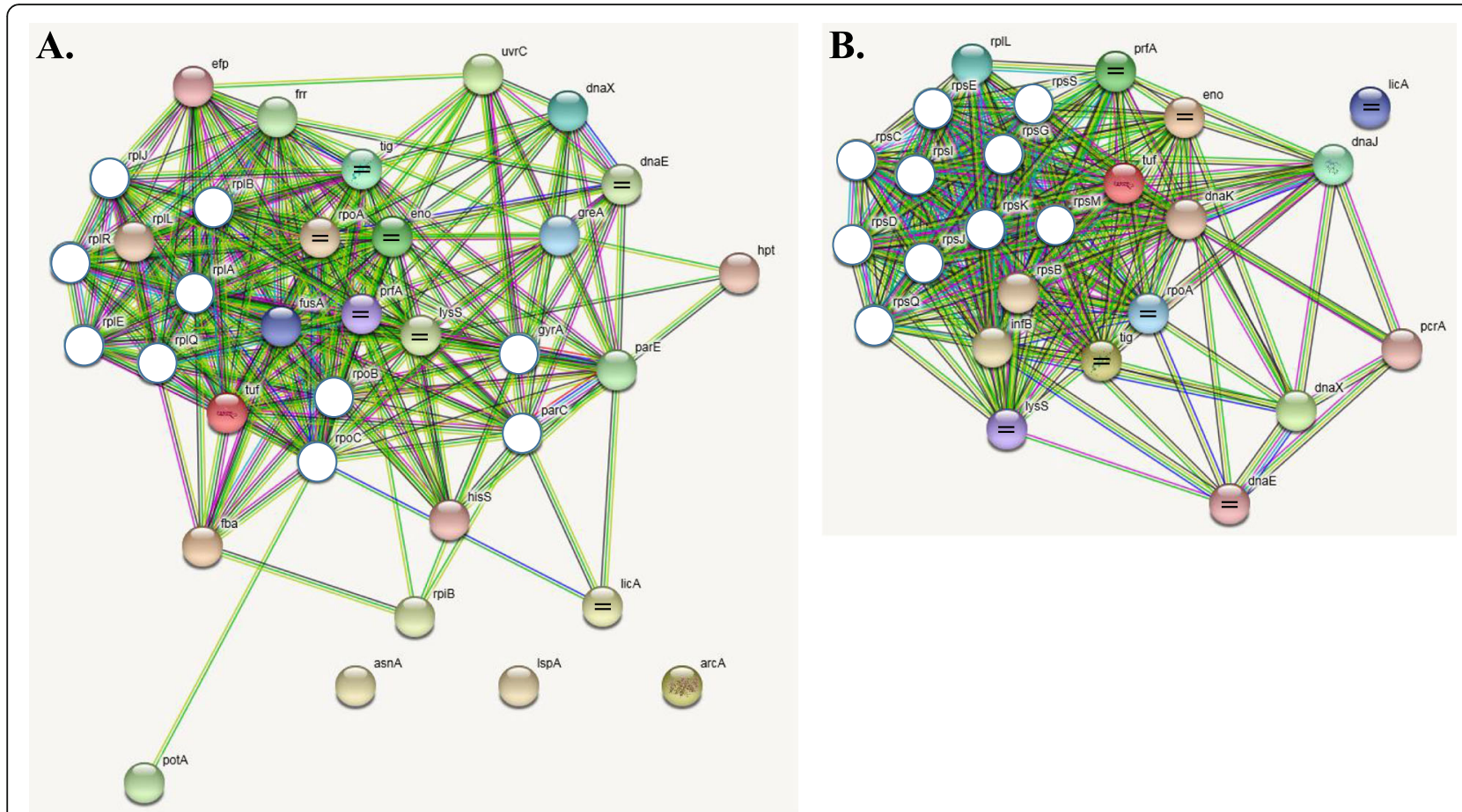

Fig. 5 The protein-protein interaction network analysis tool (STRING database) was used to analyze the proteins identified in the fractions of Mycoplasma fermentans MFI cultured in anaerobic (a) and aerobic (b) conditions. A network was build based on medium confidence (0.4) evidence. Proteins are indicated by nodes labeled with the gene symbol. The network was enriched in interactions, using the intersection of 10 additional proteins that were labeled as white nodes in the figure. Mycoplasma hominis was used as reference genome. The proteins labeled with the symbol ' $=$ ' are present both in anaerobiosis and aerobiosis. a: represents the fraction of MFI cultured in anaerobic conditions showing 246 interactions between individual proteins (or nodes). In the analysis, STRING did not include the following proteins: argF, malL, xfp, p37 and pmpr (exclusive of anaerobic MFI) abca, ptsl, pdhA, nrnA and hysA (commonly present in both anaerobic and aerobic MFI). b: shows the fraction of MFI cultured in aerobic conditions depicting 212 interactions between individual proteins. In the analysis, STRING did not include the following proteins: had, kdul, ndt, rlmb and pstb (exclusive of aerobic MFI), abca, ptsl, pdhA, nrnA, and hys (commonly present in both anaerobic and aerobic MFI)

infection with Mycoplasma fermentans subtype incognitus induces chromosomal alterations in vitro that result in phenotypic changes leading to acquisition of malignant properties in mouse and human cells, including loss of anchorage dependency, ability to form colonies in soft agar, and tumorigenicity in nude mice [56-58]. Infections with several mycoplasmas (fermentans, arginini, hominis and arthritidis) inhibit p53 activity and cooperate with Ras in oncogenic transformation in vitro, though the responsible bacterial protein has not been identified [59]. Also, mycoplasmas infections dysregulate gene expression profiles and post-translation modification in several cell lines [60]. These findings indicate that, in some cases, mycoplasmas could facilitate tumorigenesis, though (as mentioned above) no direct carcinogenic role for any mycoplasmas has been demonstrated in vivo. We isolated and characterized a strain of human mycoplasma able to induce lymphoma in a Severe Combined Immuno-Deficient (SCID) mouse model, consistent with a previously described lymphomagenesis dependent upon reduced p53 activity [40]. We demonstrated that this mycoplasma's DnaK, belonging to the HSP70 chaperone family, binds to human PARP1 and reduces its catalytic activity. PARP1 activates and recruits to the site of DNA damage important components of the DNA-repair complex. Moreover, this DnaK also binds human USP10 (ubiquitin carboxyl-terminal hydrolase 10, an important regulator of p53 stability), reducing p53 stability and anticancer functions. This indicates that, in cells where the DnaK is present, PARP1 and p53 anti-cancer activities will be reduced, increasing the likelihood of DNA instability and consequent malignant transformation. Mycoplasma was abundantly detected early in infected mice, but only low copy numbers of mycoplasma DnaK DNA sequences were found in primary and secondary tumors, suggesting a "hit and run/ hide" mechanism of transformation, in which the critical events have occurred previous to cancer detection [40].

In addition, mycoplasmas have been associated with infectious diseases and post-infection pathologies both in humans and animals, and frequently persist also as chronic, asymptomatic infections [24-27, 61], but their 
Table 4 Characteristics of biological process, molecular function and KEGG pathways of protein-protein interaction of anaerobic and aerobic Mycoplasma Fermentans with STRING 10.0

\begin{tabular}{|c|c|c|}
\hline \multicolumn{3}{|c|}{ A. MFI Anaerobic } \\
\hline \multicolumn{3}{|c|}{ Biological Process (GO) } \\
\hline Pathway ID & Pathway description & matching protein \\
\hline G0.0044267 & cellular protein metabolic process & arcA,lysS,rplB,rplQ,tuf \\
\hline GO.1901566 & organonitrogen compound biosynthetic process & $\operatorname{arcA},|y s S, r p| B, r p \mid Q, t u f$ \\
\hline G0.0006412 & translation & lysS,rp|B,rplQ,tuf \\
\hline G0.0044249 & cellular biosynthetic process & $\operatorname{arcA},|y s S, r p| B, r p \mid Q, t u f$ \\
\hline G0.0010467 & gene expression & lysS,rp|B,rplQ,tuf \\
\hline G0.0008152 & metabolic process & arcA,licA,lysS,rp|B,rplQ,tuf \\
\hline G0.0006520 & cellular amino acid metabolic process & arcA,lysS \\
\hline \multicolumn{3}{|c|}{ Molecular Function (GO) } \\
\hline G0.0003674 & molecular_function & arcA,licA,lysS,rp|B,rplQ,tuf \\
\hline G0.0003824 & catalytic activity & arcA,licA,lysS,rplB,tuf \\
\hline \multicolumn{3}{|c|}{ KEGG Pathways } \\
\hline 03020 & RNA polymerase & rpoA,rpoB,rpoC \\
\hline 00230 & Purine metabolism & dnaE,dnaX,hpt,rpoA,rpoB,rpoC \\
\hline 01110 & Biosynthesis of secondary metabolites & arcA,asnA,eno,fba,hpt,rpiB \\
\hline 01100 & Metabolic pathways & $\operatorname{arcA}$,asnA,dnaE,dnaX,eno,fba,hpt,rpiB,rpoA,rpoB,rpoC \\
\hline \multicolumn{3}{|c|}{ B. MFI Aerobic } \\
\hline \multicolumn{3}{|c|}{ Biological Process (GO) } \\
\hline Pathway ID & Pathway description & matching protein \\
\hline GO:0044267 & cellular protein metabolic process & dnaK,lysS,rpsS,tuf \\
\hline GO:0008152 & metabolic process & dnaK,licA,lysS,rpsS,tuf \\
\hline GO:0006412 & translation & lysS,rpsS,tuf \\
\hline GO:0010467 & gene expression & lysS,rpsS,tuf \\
\hline \multicolumn{3}{|c|}{ Molecular Function (GO) } \\
\hline GO:0003674 & molecular_function & dnaK,licA,lysS,rpsS,tuf \\
\hline GO:0003676 & nucleic acid binding & lysS,rpsS,tuf \\
\hline GO:0032550 & purine ribonucleoside binding & dnak,lysS,tuf \\
\hline GO:0032555 & purine ribonucleotide binding & dnak,lysS,tuf \\
\hline GO:0035639 & purine ribonucleoside triphosphate binding & dnak,lysS,tuf \\
\hline GO:0097159 & organic cyclic compound binding & dnaK,lysS,rpsS,tuf \\
\hline GO:1901363 & heterocyclic compound binding & dnak,lysS,rpsS,tuf \\
\hline GO:0005524 & ATP binding & dnak,lysS \\
\hline GO:0003723 & RNA binding & rpsS,tuf \\
\hline \multicolumn{3}{|c|}{ KEGG Pathways } \\
\hline 03010 & Ribosome & rplL, rpsB, rpsC, rpsD, rpsE, rpsG, rpsl, rpsJ, rpsK, rpsM, rspQ, rpsS \\
\hline 03430 & Mismatch Repair & dnaX, dnaE, pcrA \\
\hline 00230 & Purine metabolism & dnaE,dnaX, rpoA \\
\hline
\end{tabular}

pathogenic mechanism(s) are not completely clear yet. In this regard, it is well known that mycoplasma can evade the immune-response by modulating the immune-system through a series of mechanisms, including the interaction of membrane lipid proteins with monocyte/macrophages
[32, 62, 63]. Moreover mycoplasmas, like other bacteria, can be aerobic or facultative anaerobic microorganisms [21]. The biological determinants linked to the ability of growing in anaerobic or aerobic conditions, which is usually associated with localized infections [33, 34], have 
never been shown in Mycoplasma fermentans. It is thus of interest to better characterize the mycoplasma proteins expressed in two different growth settings mimicking aerobic and anaerobic conditions observed in vivo [64].

A number of studies have addressed the correlation between protein expression and pathogenic potential of mycoplasmas by using proteomic analysis [65] both in human (such as Mycoplasma pneumoniae [66, 67], Mycoplasma genitalium [68, 69] and Mycoplasma fermentans M64 [12]) and in animals (more in details Mycoplasma mobile that infects fishes [70], and Mycoplasma hypopneumoniae and Mycoplasma flocculare that are pathogenic for swines [71, 72]).

Our findings show a high protein ratio of anaerobic versus aerobic when the liposoluble fraction was compared to the hydrosoluble (Fig. 3). Most of the lipoproteins of mycoplasmas are believed to be exposed to the extracellular surface and have been characterized to have roles in adherence, transport of nutrients and enzymatic activity. In addition, mycoplasma lipoproteins can be related to virulence and antigenicity $[13,14]$. While many of the predicted lipoproteins of mycoplasmas remain hypothetical, our results suggest that the higher ratio of lipoproteins in the anaerobic mycoplasma might be associated to a more pathogenic phenotype. An example is the observed higher ratio of the p37 lipoprotein in the anaerobic fraction (Table 3).

By using the PANTHER software, proteins from each of the two growing conditions were classified depending on protein class and molecular functions. According to the class protein classification, our results show that proteins with isomerase activity were only found in mycoplasma grown in anaerobic conditions (Fig. 4a-b). In addition, the molecular function classification indicated that the anaerobic mycoplasma is enriched in proteins with translation regulatory activity, while more proteins with structural activity were expressed in the aerobic mycoplasma (Fig. 4cd). However, a major limitation of the PANTHER analysis was that numerous proteins from Mycoplasma fermentans were not found in the reference genome of Mycoplasma genitalium (see legend Additional file 1: Table S1). Nevertheless, we were able to predict metabolic pathways with the STRING database (Fig. 5 and Table 4) and show that mycoplasma's growing conditions affect specific metabolic pathways (Table 4). In fact, while the KEGG pathway of purine metabolism was upregulated in both growing conditions, the anaerobic MFI was enriched in metabolic pathways, biosynthesis of secondary metabolites and the RNA polymerase pathways. In contrast, the ribosome and the mismatch repair pathways were upregulated in the aerobic MFI.

Metabolic activities are important determinants for Mollicutes taxonomy, and a number of studies highlighted the metabolism of mycoplasmas by linking the enzymatic activities detected in the mollicutes (for a review see [73]) Our study provides a description of metabolic changes reflecting the ability of mycoplasma to "switch" between two opposite growing conditions, aerobic and anaerobic. This would allow the microorganism to survive and replicate, and in turn to enhance the establishment of a proinflammatory environment in the host.

It is important to consider that our method only display highly expressed proteins in the two growth conditions of MFI, therefore emphasizing upregulation of different pathways. Nevertheless, common metabolic pathways for MFI aerobic and anaerobic, such as glycolysis, are expected to be in place, as suggested by the presence of the enolase (eno) and pyruvate dehydrogenase (pdhA) in both isolates (Tables 1 and 2). Though we show important differences between aerobic and anaerobic Mycoplasma associated to metabolic requirements, further studies are needed to better characterize all the stages of these processes.

\section{Conclusions}

The identification and characterization of virulence factors is of upmost relevance to discover new targets for the development of diagnostic methods, therapeutic drugs, and vaccines. For example, studies of other microorganisms' proteasome have revealed the presence of proteins like adhesins, transporters, nucleases, bearing virulence-related functional domains. Overall, these previous studies indicate the necessity of further and more comprehensive comparative proteomic studies, to deeply investigate possible pathogenicity or virulence-related differences at the protein level.

Our proteomic analysis shows the occurrence of phenotypical changes in mycoplasma due to oxygen availability. Such changes, associated to specific metabolic processes and molecular functions, are likely to be important for the survival of the micro-organism in two different conditions (aerobic and anaerobic), and eventually to continuously induce proinflammatory cytokines and other metabolites harmful to the host. Further studies are needed to better characterize all the steps of these processes, which could be correlated to immune-evasion and pathogenesis $[74,75]$.

\section{Supplementary information}

Supplementary information accompanies this paper at https://doi.org/10. 1186/s41231-019-0047-2.

Additional file 1: Table S1. List of proteins identified in the fractions of Mycoplasma fermentans MFI cultured in anaerobic and aerobic conditions classified according to the GO annotations for protein classes and molecular functions using PANTHER software.

\section{Abbreviations}

2-DE: two-dimensional gel electrophoresis; Ae: aerobic; An: anaerobic; GO: Gene Ontology; MFI: Mycoplasma fermentans MFI 


\section{Acknowledgements}

Not applicable.

\section{Authors' contributions}

FB and SC designed and performed experiments, analyzed data, and wrote the manuscript; SK and FC helped to perform the experiments and analyzed data; HT collected and analyzed data; RCG and DZ contributed to the design of the experiments and to the writing of the manuscript. All authors read and approved the final manuscript.

\section{Funding}

This work was supported in part by a grant from the CRF Program, University of Maryland.

\section{Availability of data and materials}

All data generated or analyzed during this study are included in this published article.

\section{Ethics approval and consent to participate} Not applicable.

\section{Consent for publication}

Not applicable.

\section{Competing interests}

The authors declare that they have no competing interests.

\section{Author details}

${ }^{1}$ Institute of Human Virology, School of Medicine, University of Maryland, Baltimore, MD 21201, USA. ${ }^{2}$ Department of Biochemistry and Molecular Biology, School of Medicine, University of Maryland, Baltimore 21201, USA. ${ }^{3}$ Department of Medicine, School of Medicine, University of Maryland, Baltimore 21201, USA. Institute for Genome Sciences, School of Medicine, University of Maryland, Baltimore 21201, USA. ${ }^{5}$ Department of Microbiology and Immunology, School of Medicine, University of Maryland, Baltimore 21201, USA.

Received: 6 June 2019 Accepted: 20 September 2019

\section{Published online: 17 October 2019}

\section{References}

1. Bove JM. Molecular features of mollicutes. Clin Infect Dis. 1993;17(Suppl 1): S10-31.

2. Razin S. Peculiar properties of mycoplasmas: the smallest self-replicating prokaryotes. FEMS Microbiol Lett. 1992;100(1-3):423-31.

3. Shu HW, et al. Genome sequence of the repetitive-sequence-rich mycoplasma fermentans strain M64. J Bacteriol. 2011;193(16):4302-3.

4. Dybvig K, Voelker LL. Molecular biology of mycoplasmas. Annu Rev Microbiol. 1996;50:25-57.

5. Rechnitzer $\mathrm{H}$, et al. Genomic features and insights into the biology of mycoplasma fermentans. Microbiology. 2011;157(Pt 3):760-73.

6. Kandavelmani A, Piramanayagam S. Comparative genomics of < i $>$ mycoplasma $</ i>$ : insights on genome reduction and identification of potential antibacterial targets. Biomed Biotechnol Res J. 2019;3(1):9-18.

7. Baseman JB, Tully JG. Mycoplasmas: sophisticated, reemerging, and burdened by their notoriety. Emerg Infect Dis. 1997;3(1):21-32.

8. Shimizu T, Kida Y, Kuwano K. Lipid-associated membrane proteins of mycoplasma fermentans and M. penetrans activate human immunodeficiency virus long-terminal repeats through toll-like receptors. Immunology. 2004;113(1):121-9.

9. Fujita $\mathrm{M}$, et al. Involvement of leucine residues at positions 107, 112, and 115 in a leucine-rich repeat motif of human toll-like receptor 2 in the recognition of diacylated lipoproteins and lipopeptides and Staphylococcus aureus peptidoglycans. J Immunol. 2003;171(7):3675-83.

10. Takeda K, Takeuchi O, Akira S. Recognition of lipopeptides by toll-like receptors. J Endotoxin Res. 2002;8(6):459-63.

11. Gerlic M, et al. The inhibitory effect of mycoplasma fermentans on tumour necrosis factor (TNF)-alpha-induced apoptosis resides in the membrane lipoproteins. Cell Microbiol. 2007;9(1):142-53.
12. Liu YC, et al. Proteomics characterization of cytoplasmic and lipid-associated membrane proteins of human pathogen mycoplasma fermentans M64. PLoS One. 2012;7(4):e35304.

13. Christodoulides A, et al. The role of lipoproteins in mycoplasma-mediated immunomodulation. Front Microbiol. 2018;9:1682.

14. Browning GF, et al. The central role of lipoproteins in the pathogenesis of mycoplasmoses. Vet Microbiol. 2011;153(1-2):44-50.

15. Yavlovich $\mathrm{A}$, et al. The reducing antioxidant capacity of mycoplasma fermentans. FEMS Microbiol Lett. 2006;259(2):195-200.

16. Lo SC, et al. Adhesion onto and invasion into mammalian cells by mycoplasma penetrans: a newly isolated mycoplasma from patients with AIDS. Mod Pathol. 1993;6(3):276-80.

17. Hegde $\mathrm{S}$, et al. In vitro and in vivo cell invasion and systemic spreading of mycoplasma agalactiae in the sheep infection model. Int J Med Microbiol. 2014:304(8):1024-31.

18. Baseman JB, et al. Interplay between mycoplasmas and host target cells. Microb Pathog. 1995:19(2):105-16.

19. Yavlovich A, et al. Mycoplasma fermentans binds to and invades HeLa cells: involvement of plasminogen and urokinase. Infect Immun. 2004; 72(9):5004-11.

20. Benedetti $\mathrm{F}$, et al. Anti-inflammatory effects of $\mathrm{H} 2 \mathrm{~S}$ during acute bacterial infection: a review. J Transl Med. 2017;15(1):100.

21. Razin S, Yogev D, Naot Y. Molecular biology and pathogenicity of mycoplasmas. Microbiol Mol Biol Rev. 1998;62(4):1094-156.

22. Wade WG. The oral microbiome in health and disease. Pharmacol Res. 2013 69(1):137-43.

23. Taylor-Robinson D. Mollicutes in vaginal microbiology: mycoplasma hominis, Ureaplasma urealyticum, Ureaplasma parvum and mycoplasma genitalium. Res Microbiol. 2017;168(9-10):875-81.

24. Yanez $\mathrm{A}$, et al. Animal model of mycoplasma fermentans respiratory infection. BMC Res Notes. 2013;6:9.

25. He J, et al. [Corrigendum] Insights into the pathogenesis of Mycoplasma pneumoniae (Review). Mol Med Rep. 2018;17(3):4155

26. Taylor-Robinson D, Furr PM. Models of infection due to mycoplasmas, including mycoplasma fermentans, in the genital tract and other sites in mice. Clin Infect Dis. 1993:17(Suppl 1):S280-2.

27. Tamarelle J, et al. The vaginal microbiota and its association with human papillomavirus, chlamydia trachomatis, Neisseria gonorrhoeae and mycoplasma genitalium infections: a systematic review and meta-analysis. Clin Microbiol Infect. 2019;25(1):35-47.

28. Tully JG, et al. A newly discovered mycoplasma in the human urogenital tract. Lancet. 1981;1(8233):1288-91.

29. Gilroy CB, Keat A, Taylor-Robinson D. The prevalence of mycoplasma fermentans in patients with inflammatory arthritides. Rheumatology (Oxford). 2001:40(12):1355-8.

30. Horowitz $\mathrm{S}$, et al. Mycoplasma fermentans in rheumatoid arthritis and other inflammatory arthritides. J Rheumatol. 2000:27(12):2747-53.

31. Gil C, et al. Presence of mycoplasma fermentans in the bloodstream of Mexican patients with rheumatoid arthritis and IgM and lgG antibodies against whole microorganism. BMC Musculoskelet Disord. 2009;10:97.

32. Rottem S. Interaction of mycoplasmas with host cells. Physiol Rev. 2003; 83(2):417-32.

33. Hentges DJ. Anaerobes: General Characteristics. In: Baron S, editor. Medica Microbiology, 4th edition. University of Texas Medical Branch at Galveston (TX); 1996.

34. Noor A, Bhimji SS. Anaerobic Infections. In: StatPearls. Treasure Island; 2018.

35. Yamaguchi $M$, et al. Abscess formation due to mycoplasma hominis infection after cesarean section. J Obstet Gynaecol Res. 2009;35(3):593-6.

36. Mori $\mathrm{N}$, et al. Pelvic abscess due to mycoplasma hominis following caesarean section. JMM Case Rep. 2016;3(4):e005059.

37. Koshiba $\mathrm{H}$, et al. Hematoma and abscess formation caused by mycoplasma hominis following cesarean section. Int J Women's Health. 2011:3:15-8.

38. Kennedy KJ, Prince S, Makeham T. Mycoplasma hominis-associated parapharyngeal abscess following acute Epstein-Barr virus infection in a previously immunocompetent adult. J Clin Microbiol. 2009;47(9):3050-2.

39. Parsonson F. Mycoplasma hominis infection following neurosurgical intervention in a patient with spinal cord compression. JMM Case Rep. 2016:3(2):1-4.

40. Zella D, et al. Mycoplasma promotes malignant transformation in vivo, and its DnaK, a bacterial chaperone protein, has broad oncogenic properties. Proc Natl Acad Sci. 2018;115(51):E12005-14. 
41. Shu HW, et al. Complexity of the mycoplasma fermentans M64 genome and metabolic essentiality and diversity among mycoplasmas. PLoS One. 2012;7(4):e32940.

42. Ozcan SA, Miles R. Biochemical diversity of mycoplasma fermentans strains. FEMS Microbiol Lett. 1999;176(1):177-81

43. Bordier C. Phase separation of integral membrane proteins in triton X-114 solution. J Biol Chem. 1981;256(4):1604-7.

44. Shevchenko $\mathrm{G}$, et al. Cloud-point extraction and delipidation of porcine brain proteins in combination with bottom-up mass spectrometry approaches for proteome analysis. J Proteome Res. 2010;9(8):3903-11.

45. Wessel D, Flugge UI. A method for the quantitative recovery of protein in dilute solution in the presence of detergents and lipids. Anal Biochem. 1984;138(1):141-3.

46. Tshala-Katumbay D, et al. Probing mechanisms of Axonopathy. Part I: protein targets of 1,2-Diacetylbenzene, the neurotoxic metabolite of aromatic solvent 1,2-Diethylbenzene. Toxicol Sci. 2008;105(1):134-41.

47. Conesa A, et al. Blast2GO: a universal tool for annotation, visualization and analysis in functional genomics research. Bioinformatics. 2005; 21(18):3674-6.

48. Thomas PD, et al. PANTHER: a library of protein families and subfamilies indexed by function. Genome Res. 2003;13(9):2129-41.

49. $\mathrm{Mi} \mathrm{H}$, et al. Large-scale gene function analysis with the PANTHER classification system. Nat Protoc. 2013;8(8):1551-66.

50. Szklarczyk D, et al. STRING v10: protein-protein interaction networks, integrated over the tree of life. Nucleic Acids Res. 2015;43(Database issue): D447-52.

51. Park DM, et al. The bacterial response regulator ArcA uses a diverse binding site architecture to regulate carbon oxidation globally. PLoS Genet. 2013; 9(10):e1003839.

52. Gong $M$, et al. p37 from mycoplasma hyorhinis promotes cancer cell invasiveness and metastasis through activation of MMP-2 and followed by phosphorylation of EGFR. Mol Cancer Ther. 2008;7(3):530-7.

53. Barykova YA, et al. Association of Mycoplasma hominis infection with prostate cancer. Oncotarget. 2011;2(4):289-97.

54. Henrich B, et al. Mycoplasma salivarium as a dominant coloniser of Fanconi anaemia associated oral carcinoma. PLoS One. 2014;9(3):e92297.

55. Ainsworth JG, et al. An association of disseminated mycoplasma fermentans in HIV-1 positive patients with non-Hodgkin's lymphoma. Int J STD AIDS. 2001;12(8):499-504.

56. Jiang $\mathrm{S}$, et al. Mycoplasma infection transforms normal lung cells and induces bone morphogenetic protein 2 expression by post-transcriptional mechanisms. J Cell Biochem. 2008;104(2):580-94.

57. Namiki $K$, et al. Persistent exposure to mycoplasma induces malignant transformation of human prostate cells. PLoS One. 2009;4(9):e6872.

58. Zhang S, Tsai S, Lo S-C. Alteration of gene expression profiles during mycoplasma-induced malignant cell transformation. BMC Cancer. 2006;6:116.

59. Logunov DY, et al. Mycoplasma infection suppresses p53, activates NF-KB and cooperates with oncogenic Ras in rodent fibroblast transformation. Oncogene. 2008;27(33):4521-31.

60. Liu W, Shou C. Mycoplasma hyorhinis and mycoplasma fermentans induce cell apoptosis and changes in gene expression profiles of 32D cells. Biol Res. 2011;44(4):383-91.

61. Kawahito $Y$, et al. Mycoplasma fermentans glycolipid-antigen as a pathogen of rheumatoid arthritis. Biochem Biophys Res Commun. 2008;369(2):561-6.

62. Rawadi G. Mycoplasma fermentans interaction with monocytes/ macrophages: molecular basis. Microbes Infect. 2000;2(8):955-64.

63. Seya T, Matsumoto M. A lipoprotein family from mycoplasma fermentans confers host immune activation through toll-like receptor 2. Int J Biochem Cell Biol. 2002;34(8):901-6.

64. Wenger $\mathrm{RH}$, et al. Frequently asked questions in hypoxia research. Hypoxia (Auckl). 2015;3:35-43.

65. Jaffe JD, Berg HC, Church GM. Proteogenomic mapping as a complementary method to perform genome annotation. Proteomics. 2004; 4(1):59-77.

66. Catrein I, Herrmann R. The proteome of mycoplasma pneumoniae, a supposedly "simple" cell. Proteomics. 2011;11(18):3614-32.

67. Regula JT, et al. Defining the mycoplasma 'cytoskeleton': the protein composition of the triton $\mathrm{X}-100$ insoluble fraction of the bacterium mycoplasma pneumoniae determined by 2-D gel electrophoresis and mass spectrometry. Microbiology. 2001;147(Pt 4):1045-57.
68. Balasubramanian S, et al. Proteomics of mycoplasma genitalium: identification and characterization of unannotated and atypical proteins in a small model genome. Nucleic Acids Res. 2000;28(16):3075-82.

69. Parraga-Nino $\mathrm{N}$, et al. A comprehensive proteome of mycoplasma genitalium. J Proteome Res. 2012;11(6):3305-16.

70. Jaffe JD, et al. The complete genome and proteome of mycoplasma mobile. Genome Res. 2004;14(8):1447-61.

71. Paes JA, et al. Comparative proteomics of two mycoplasma hyopneumoniae strains and mycoplasma flocculare identified potential porcine enzootic pneumonia determinants. Virulence. 2018;9(1):1230-46.

72. Pinto PM, et al. Comparative proteomic analysis of pathogenic and nonpathogenic strains from the swine pathogen mycoplasma hyopneumoniae. Proteome Sci. 2009:7:45.

73. Dennis, Pollack J, Williams M, McElhaney RN. The Comparative Metabolism of the Mollicutes (Mycoplasmas ): The Utility for Taxonomic Classification and the Relationship of Putative Gene Annotation and Phylogeny to Enzymatic Function in the Smallest Free-Living Cells. Crit Rev Microbiol. 1997;23(4):269-354

74. Gautier-Bouchardon AV. Antimicrobial Resistance in Mycoplasma spp. Microbiol Spectr. 2018;6(4). https://doi.org/10.1128/microbiolspec.ARBA0030-2018.

75. Zheng X, et al. Macrolide-Resistant Mycoplasma pneumoniae, United States. Emerg Infect Dis. 2015;21(8):1470-2.

\section{Publisher's Note}

Springer Nature remains neutral with regard to jurisdictional claims in published maps and institutional affiliations.
Ready to submit your research? Choose BMC and benefit from:

- fast, convenient online submission

- thorough peer review by experienced researchers in your field

- rapid publication on acceptance

- support for research data, including large and complex data types

- gold Open Access which fosters wider collaboration and increased citations

- maximum visibility for your research: over $100 \mathrm{M}$ website views per year

At $\mathrm{BMC}$, research is always in progress.

Learn more biomedcentral.com/submissions 